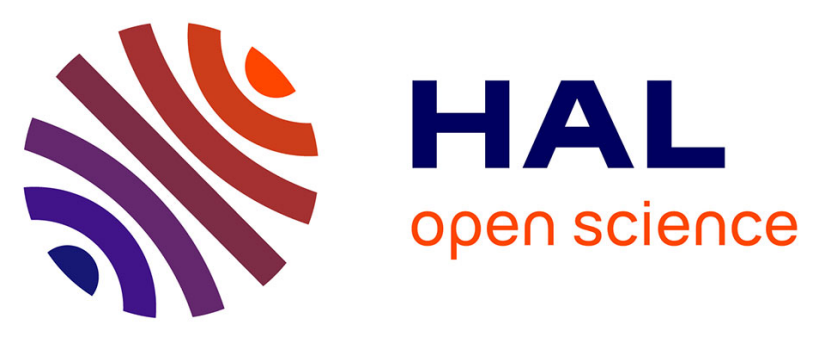

\title{
Evaluation of an IEEE 802.11n Communication System in presence of Transient Electromagnetic Interferences from the Pantograph-Catenary Contact
}

Grecia Romero, Eric Pierre Simon, Virginie Deniau, Christophe Gransart, Mohamed Kousri

\section{- To cite this version:}

Grecia Romero, Eric Pierre Simon, Virginie Deniau, Christophe Gransart, Mohamed Kousri. Evaluation of an IEEE 802.11n Communication System in presence of Transient Electromagnetic Interferences from the Pantograph-Catenary Contact. XXXII General Assembly and Scientific Symposium of the International Union of Radio Science, Montreal, 19-26 August 2017, General Assembly and Scientific Symposium of the International Union of Radio Science, Aug 2017, Montreal, Canada. hal-01684681

\section{HAL Id: hal-01684681 \\ https://hal.science/hal-01684681}

Submitted on 15 Jan 2018

HAL is a multi-disciplinary open access archive for the deposit and dissemination of scientific research documents, whether they are published or not. The documents may come from teaching and research institutions in France or abroad, or from public or private research centers.
L'archive ouverte pluridisciplinaire HAL, est destinée au dépôt et à la diffusion de documents scientifiques de niveau recherche, publiés ou non, émanant des établissements d'enseignement et de recherche français ou étrangers, des laboratoires publics ou privés. 


\title{
Evaluation of an IEEE 802.11n Communication System in presence of Transient Electromagnetic Interferences from the Pantograph-Catenary Contact
}

\author{
Grecia Romero*(1) $^{(1)}$ Eric Pierre Simon ${ }^{(1)}$, Virginie Deniau ${ }^{(2)}$, Christophe Gransart ${ }^{(2)}$ and Mohamed Kousri ${ }^{(2)}$ \\ (1) University of Lille, Villeneuve d'Ascq, France grecia.romero@ed.univ-lille1.fr*, eric.simon@univ-lille1.fr \\ (2) IFSTTAR,Villeneuve d'Ascq, France,virginie.deniau@ifsttar.fr, christophe.gransart@ifsttar.fr, mohamed.kousri@ifsttar.fr
}

\begin{abstract}
This paper presents a susceptibility study of an IEEE $802.11 \mathrm{n}$ communication network in an electromagnetic (EM) environment representative of the railway EM environment, which is characterized by the presence of transient EM interferences induced by the sliding contact between the pantograph and the catenary. In Europe, the ground-train communications are currently carried out by the GSM-R communication system, but the next generation of ground-train radio which could be based on OFDM solutions is already discussed. Then, we worked on the susceptibility of OFDM communication signals to the EM transient interferences which are systematically present in the railway EM environment. To achieve this susceptibility analysis, we select an IEEE 802.11 n communication network and we analyzed its performances in the presence of transient EM interferences. The transient interferences present in the railway environment, were characterized in the literature as signals of short duration, variable period and broadband. Accordingly, the time interval between the successive transient signals and their time duration were used as main characteristics in order to study their impact on the achieved bit rate of the communications. A set of measurements was performed within a controlled environment to evaluate the performance of the IEEE $802.11 \mathrm{n}$ communication network in presence of such interference signals.
\end{abstract}

\section{Introduction}

Nowadays, the GSM-R (global system for mobile communication dedicated to railway) is the main standard in Europe to exchange voice communication, signaling information and control data between trains and control centers. However, the limitations of the GSM-R in relation to more recent communication solutions are under discussion in order to develop the next generation of ground-train radio communication. Moreover, to meet the expectations of its passengers and remain attractive, the railway sector must evolve and offer access to the Internet in the trains. As a consequence, new communication technologies based on Orthogonal Frequency Division Multiplexing (OFDM) signals could be employed for railway operational applications or passenger services, including Internet access. However, the railway electromagnetic (EM) environment is characterized by various electromagnetic interferences which can affect the communications. Several studies [1, 2, 3] have been carried out regarding transient EM interferences generated by the contact discontinuities between the pantograph and the catenary. These very short time interferences are broadband interferences which can cover the Wi-Fi frequency bands. Therefore, in this paper, we study the susceptibility of an OFDM communication system face to the transient EM interferences representative of those produced by the catenary-pantograph sliding contact. There are many wireless communication standards available that could be used for Wi-Fi service in railway. One of them is the IEEE 802.11n standard [4] which is based on OFDM signals. We then studied its susceptibility in presence of transient EM interferences in order to identify the limits of such standard for applications on board trains. The rest of the paper is organized as follows. In Section 2, first, we describe the transient electromagnetic signal used for the experimental tests and we detail the whole experimental setup. In Section 3, we present and analyze the measurement results. Finally, we conclude in Section 4.

\section{Experimental method}

In this section, we describe the interference signal and the test setup used to assess the impact of the transient EM interferences on IEEE 802.11n networks.

\subsection{Transient EM interferences signal char- acteristics}

For this study, we generate an interference signal with an arbitrary waveform generator (AWG) and make vary the repetition period $T$ of the interference signal. We have to use an interference signal waveform that is representative of those observed on a train but also appropriate to analyze the impact of the different interference characteristics. Previous studies [5] mentioned that the transient interferences produced by the losses of contact between the catenary and the pantograph are double exponential signals. However, the use of double exponential signals is not suited to a parametric study because it can be strongly deformed by amplification or attenuation. Moreover, to be able to analyze the 
results, it is important that the interference signal covers the entire $2.4 \mathrm{GHz}-2.5 \mathrm{GHz} \mathrm{Wi}-\mathrm{Fi}$ band in a homogeneous way.

Knowing that a double exponential signal can be approximated as a sum of sinusoidal signals with a zero shift phase at the time $t=0$, by modifying the phase shift at $t=0$ of the sinusoidal components, we can obtain a wide band signal, which can be amplified without introducing saturation phenomena and deformation in the frequency domain. We can also control the covered frequency band by selecting the frequencies of sinusoidal components between $F_{\text {start }}=2.4 \mathrm{GHz}$ to $F_{\text {stop }}=2.5 \mathrm{GHz}$ and precisely set the time duration $D$ of the interference signal:

$$
D=N_{p} / F_{s}
$$

where $F_{s}$ is the sampling frequency of the AWG, $N_{p}$ is the number of points over the time of the impulsive signal.

The interference signal is then designed from a sum of cosines with a random phase $\left(\varphi_{n}\right)$ at $t=0$ and defined by:

$$
i(t)=\sum_{n=N \min }^{N \max } A \cdot \cos \left(2 \pi n F_{\text {step }} t+\varphi_{n}\right),
$$

Where $A$ is magnitude of the harmonics, $F_{\text {step }}=$ $F_{S} / N_{p}$ is frequency step between the successive harmonic components, $N_{\text {min }}=$ integer $\left(F_{\text {start }} / F_{\text {step }}\right)$ and $N_{\text {max }}=$ integer $\left(F_{\text {stop }} / F_{\text {step }}\right)$.

Fig. 1 shows us the time domain and Time-Frequency representations of transient electromagnetic (EM) interferences signal with period $T=26 \mu \mathrm{s}$ and duration $D=0.8 \mu \mathrm{s}$. On the Time-Frequency representation, we can verify that the signal covers the entire $2.4 \mathrm{GHz}-2.5 \mathrm{GHz}$ Wi-Fi band.
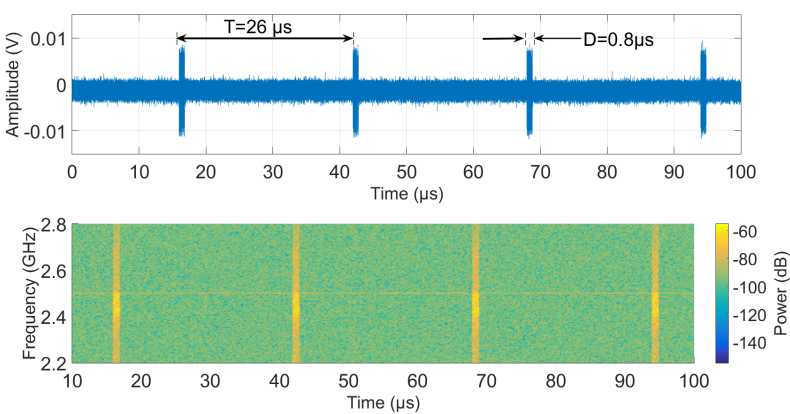

Figure 1. Time domain and Time-Frequency representations of transient EM interferences signal of $D=0.8 \mu \mathrm{s}$ generated by the AWG with a period of $T=26 \mu \mathrm{s}$.

\subsection{Test setup}

Fig. 2 presents the equipment and test setup employed. We used the AWG (Tektronix AWG7102) to generate the transient EM interference signal (section 2.1) obtained through Matlab. The generator was connected to the variable attenuation control unit in order to adjust the power of the interference signal transmitted by the antenna (horn antenna
$700 \mathrm{MHz}-18 \mathrm{GHz}$ ). On the other side, we used a monitoring system composed by an oscilloscope (LeCroy Wave Master $813 Z i$ ) with a $50 \Omega$ impedance, a sampling rate $\left(F_{S}\right)$ of $10 \mathrm{GSamples} / \mathrm{s}$ and an horn antenna $(700 \mathrm{MHz}-18 \mathrm{GHz})$, in order to measure the interference signal average power and the IEEE $802.11 \mathrm{n}$ communication average power and finally calculate the interference to signal power ratio (ISR).

The experiment was carried out in a semi-anechoic chamber at the University of Lille 1. Inside the chamber (Fig. 3), we placed the test network (a server, an access point and a client), the transmitting antenna of the interference and the monitoring antenna. Both antennas were oriented in the direction of the center of the line of sight between the client and the access point.

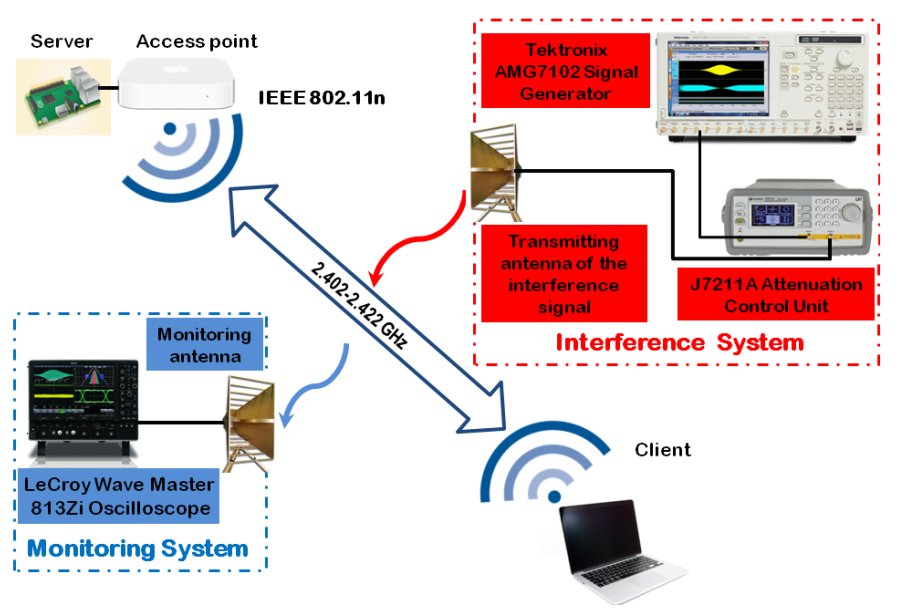

Figure 2. The test network, monitoring and interference systems.

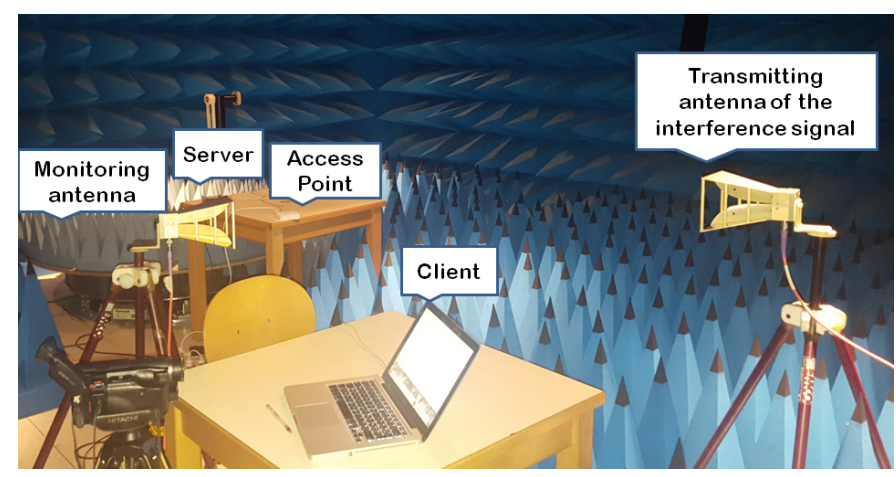

Figure 3. Experimental setup in the semi-anechoic chamber.

In order to study the transient EM interference impact originated from the pantograph-catenary subsystem on the IEEE 802.11n communication network performance, we used an interference signal with a defined duration $(D)$ and the period $(T)$ was decreased step by step, starting from $1000 \mu \mathrm{s}$ until the measurement of the bit rate reached zero, that is, the communication was interrupted. For each tested value 
of $T$, the bit rate was measured, by means of Iperf3 program [6] which was run with default settings on the client side.

Next, the bit rate measurements were repeated in making vary the interference to signal power ratio (ISR) thanks to the variable attenuation control unit. First, we used an interference signal with a defined duration $(D)$ and a constant period $(T)$. Then, the ISR was varied by increasing the interference signal power until the measurement of the bit rate reached zero.

\section{Measurement results}

Fig. 4 shows the bit rate measurements obtained for transient EM interference signals of different $D$ values $(0.8 \mu \mathrm{s}$ and $1.6 \mu \mathrm{s})$. For each $D$ value, the bit rate is given as a function of the period between consecutive transients $(T)$. We observed a varying impact of $T$ on the achieved bit rate. For a $T$ bigger than $600 \mu \mathrm{s}$ the bit rate is not affected (maximum bit rate value reached). When $T$ decreases between $500 \mu \mathrm{s}$ and $T=27 \mu \mathrm{s}$, the achieved bit rate progressively decreased. Hence, for $T=26 \mu s$ and for both $D$ values, the communication was suddenly interrupted. This interruption at $T=26 \mu \mathrm{s}$ can be explained by the MAC layer characteristics. Indeed, in the IEEE 802.11n standard [4], the channel has to be free during a minimum duration called the DCF Interframe Space (DIFS) duration $(28 \mu \mathrm{s})$ to allow the WLAN station accessing the medium and transmit the data. Thus, as soon as the interference signal is systematically repeated during this period, the results seem to show that the channel is considered busy.

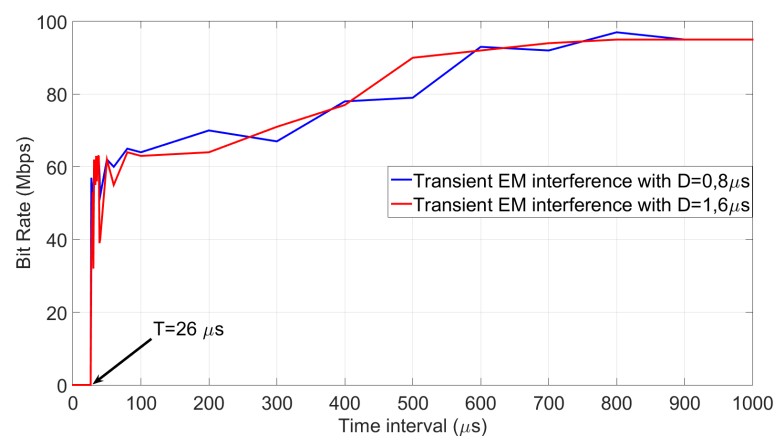

Figure 4. Bit Rate measurements for interference signals, as a function of the $\mathrm{T}$.

Now, Fig. 5 presents the achieved bit rate in presence of transient EM interference signals of $D=1.6 \mu \mathrm{s}$ with two different values of $T(26 \mu \mathrm{s}$ and $27 \mu \mathrm{s})$, in order to understand the behavior of IEEE $802.11 \mathrm{n}$ network around $26 \mu \mathrm{s}$. As can be seen, for $I S R=-10 \mathrm{~dB}$ with $T=26 \mu \mathrm{s}$ the communication is lost. Nevertheless, for $T=27 \mu \mathrm{s}$ the communication is maintained but with a lower bit rate. This result illustrates that with $T=26 \mu \mathrm{s}$ the channel is considered as busy while with a $T=27 \mu \mathrm{s}$ the channel is still available but requires a lower bit rate. Then, for $T=27 \mu \mathrm{s}$, the variation of the bit rate demonstrates that the communication system adapts the Modulation and Coding Scheme(MCS) in- dex value to the current wireless channel conditions. Each MCS index represents a set of parameters relating to the data encoding and modulation, and the maximum bit rate depends of the MCS index values.

We then observe, that with a $T=26 \mu s$, the channel is considered busy but not with a $T=27 \mu \mathrm{s}$. Nevertheless, both $T$ tested values are inferior to the $28 \mu \mathrm{s}$ DIFS duration during which the channel is sensed by MAC layer. The algorithm applied to sense the channel is called the Clear Channel Assessment (CCA). According to [4], it consists in measuring the highest average power over the channel but the method of acquisition of highest average power is not precisely described in the standard. Indeed, the standard only indicates that the CCA indicator should report that the channel is busy within a $4 \mu s$ maximum time. Therefore, to perfectly understand the difference of behavior of the communication system for $T=26 \mu s$ or $27 \mu s$, it would be necessary to examine the CCA algorithm in order to assess how the period $T$ impacts the measured highest average power over the channel.

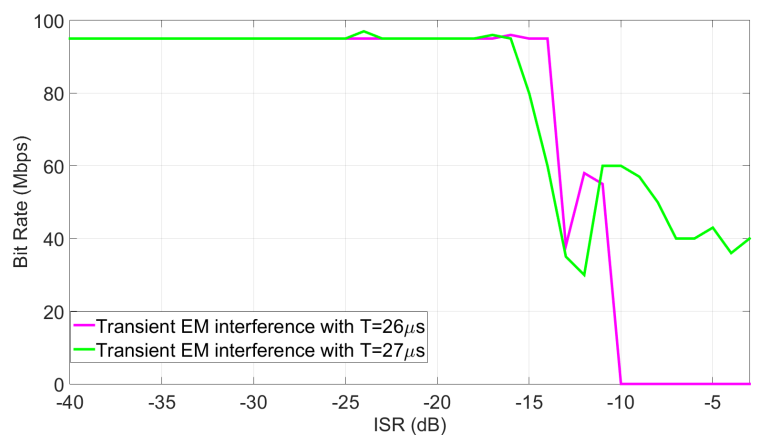

Figure 5. Bit Rate measurements of an IEEE 802.11n network under interference signals of $D=1.6 \mu \mathrm{s}$, as a function of the ISR.

Finally, we studied the impact of the duration of the interference on the communication, taking into account that the OFDM symbol duration is $3.2 \mu \mathrm{s}$ plus a $0.8 \mu \mathrm{s}$ guard interval [4]. In Fig. 6, we compare the achieved bit rate for interference signals of duration $0.8 \mu \mathrm{s}$ and $1.6 \mu \mathrm{s}$, as a function of the ISR. Then, we progressively increased the power of the interference signal and we measured the evolution of the bit rate. We notice that when $D=0.8 \mu \mathrm{s}$ an ISR of $-8 \mathrm{~dB}$ is required to lose the communication while for $D=1.6 \mu \mathrm{s}$ an ISR of $-10 \mathrm{~dB}$ is sufficient. That means that the duration of the interference impacts the highest average power measured by the CCA which reports that the channel is busy or not.

\section{Conclusion}

This paper presents a study of the performances of IEEE 802.11n communication network under transient EM interferences, which can be representative of those present in the railway environment. The analysis considered a transient EM signal which is a sum of cosines signals between 


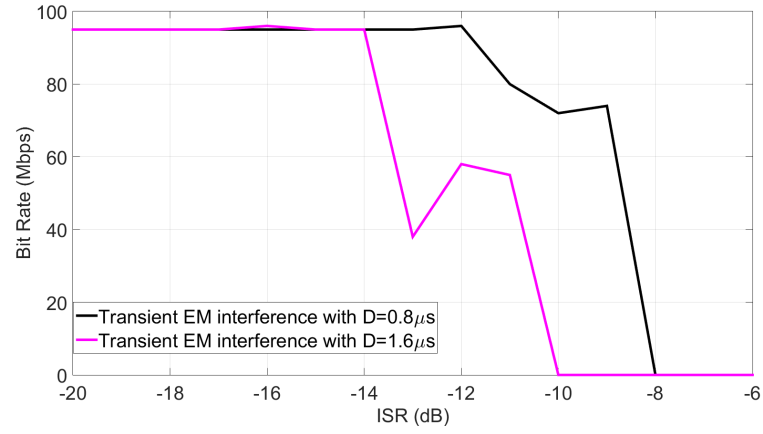

Figure 6. Bit Rate measurements of an IEEE 802.11n network under interference signals of $T=26 \mu \mathrm{s}$, as a function of the ISR.

2.4 and $2.5 \mathrm{GHz}$. We studied the impact of the time interval between consecutive transients and the impact of the duration of the transients. The experimental results allowed us to show that when the time interval is inferior to $26 \mu \mathrm{s}$, the communication system can considere the channel busy and the communication can be lost. Then, the performance of such standard in the railway environment can significantly depend on the repetition rate of the transient produced by the catenary-pantograph sliding contact. The DIFS period which is imposed by the MAC layer to sense the channel can be a limitation for applications in the railway environment if the repetition rate of transient interferences is inferior to the DIFS period. In parallel, the CCA process applied to sense the channel also consists of measuring the highest average power over the channel and this value depends on the interference power but also on the interference duration. It is then important to reduce the coupling between the interferences produced by the catenarypantograph contact and the antennas of the OFDM receivers to permit WLAN station accessing the medium.

\section{Acknowledgment}

This works has been carried out in the framework of The ELSAT2020 project which is co-financed by the European Union with the European Regional Development Fund, the French state and the Hauts de France Region Council.

\section{References}

[1] V. Deniau, J. Rioult, S. Baranowski and G. Nottet, "Measurements and post-processing for achievement of electromagnetic transient interference models: Application to the signals provided by railway substations," 2012 International Symposium on Electromagnetic Compatibility (EMC EUROPE), 17-21, September 2012, pp. 1-5.

[2] S. Chen and F. Sha, "Three Types of Electromagnetic Noise between Pantograph and Catenary," 2009 3rd IEEE International Symposium on Microwave, Antenna, Propagation and EMC Technologies for Wire- less Communications, 27-29, October 2009, pp. 4043.

[3] J. del Portillo, M. Osinalde, E. Sukia, I. Sancho, J. Mendizabal, and J. Melendez, "Characterization of the EM environment of railway spot communication systems," EMC 2008. IEEE International Symposium on Electromagnetic Compatibility, 2008 ,2008, pp. 16.

[4] "IEEE Standard for Information technologyTelecommunications and information exchange between systems Local and metropolitan area networks-Specific requirements Part 11: Wireless LAN Medium Access Control (MAC) and Physical Layer (PHY) Specifications,"IEEE Std 802.11-2012,March 2012.

[5] S. Dudoyer, R. Adriano, V. Deniau, N. N Ben Slimen, and B. Meyniel, "Testing of the GSM-R System against Electromagnetic Disturbances present in the Railway Environment," 2009 9th International Conference on Intelligent Transport Systems Telecommunications,(ITST), 20-22, October 2009 , pp. 117-122.

[6] Iperf, https://iperf.fr 